\title{
An Integrated Method to Acquire Fuzzy Rules
}

\author{
Liyan Llü, a \\ ${ }^{1}$ City Institute, Dalian University of Technology, Dalian Liaoning 116600, China \\ aemail: liuliyan81@sina.com
}

\section{Keywords: Kohonen Network; Rough Set Theory; Fuzzy Neural Network; Fuzzy Rule}

\begin{abstract}
An integrated method to acquire fuzzy rules is presented. First Kohonen network is used to quantify data, and then the rough set theory is used to produce the initial rules, and the model of fuzzy neural network is established according to the rules, to generate less concise rules, finally through the experiment, the validity and practicability of the method is verified.
\end{abstract}

\section{Introduction}

In many applications of fuzzy systems, such as fuzzy reasoning, fuzzy logic controller and fuzzy classifier, fuzzy rule extraction is a key step, but it is a very difficult problem to determine the fuzzy rules. In recent years, some scholars put forward some methods of fuzzy rules acquisition from input and output data [1-4]. The traditional method to obtain fuzzy rules is carried out according to the expert experience, usually is not accurate enough, if fully according to the experience to obtain fuzzy rules, it is very difficult to get the optimized fuzzy system.

Fuzzy neural network [5] is that fuzzy technique and neural network are combined, not only with self-learning, self-organizing, self-adaptation and fault-tolerant capability, but also has the ability of accurate approximation convergence and high precision; but the drawback is that the space dimension of input information can not be simplified, when the space dimension of input information is large, the network is not only complex, and has long training time. Through using rough set theory [6][7] not only the redundant input information can be gotten rid of, and the expressing space of input information is simplified, but also only using the data itself to provide information, not requiring a priori knowledge, so as not to need to have the perfect field expert knowledge, but the drawback is that only quantified or discrete data can be processed. Kohonen network [8] has the function of data quantification.

Based on the traditional method of obtaining fuzzy rules, Kohonen network, rough set theory and FNN are integrated, and the three advantages and disadvantages are combined and complemented each other, this paper an integrated method to acquire fuzzy rules is put forward. The proposed method has the ability of self-learning, to eliminate the false and retain the true, to eliminate rough and refinement, and to acquire fuzzy rules refining, so as to make fuzzy system more intelligent. Finally, through the example, the validity and practicability of the method is verified.

\section{Proposed Model for Acquiring Fuzzy Rules}

The basic idea to acquire fuzzy rules is: first using the Kohonen network to quantify data, and then using the indistinguishable relation and indistinguishable class and simple calculation method of the rough set theory, to find the first simplified rules from the quantified data, then establishing FNN model according to the rules, so the network has the tidy scale at the very start, at the same time the parameters of fuzzy rules can be optimized, finally based on a few simple judgment principles to further simplify the fuzzy rules.

Because only the quantified attributes can be used, when condition attribute is optimized in the rough sets theory, the continuous condition attribute quantification is the key problem of restricting the application of rough set theory. In order to solve this problem, this paper the Kohonen network is selected to quantify condition attribute, and specific algorithm is seen in reference [9]. 
In rough set theory, starting point to deal with the problem is to use the indistinguishable relation and indistinguishable class and simple calculation method of the theory to discovery the initial rules from the quantified data table. The specific steps are as follows:

The specific algorithm of attribute reduction to the compatible data table is seen in reference [10];

The attribute value reduction to the minimum attribute reduction table can make the rules simplified, and the specific algorithm of value reduction is seen in reference [11];

Then the confidence coefficient of each rule is computed, here rough membership function is made as the confidence coefficient of rules. Rough membership function is $\mu_{B}(x, X)=\left|[x]_{B} \cap X\right| /\left|[x]_{B}\right|$, and $\mid$. shows the number of elements of the collection.

In order to obtain fuzzy rules more refined, the FNN can be used to further optimize the rules; at the same time because the redundant attributes are deleted through the rough set theory, and data table is initially simplified, thus the scale of the FNN can be greatly reduced, and the training speed is improved, in order to faster convergence.

With multiple input and single output fuzzy system as an example, the fuzzy rules are used in the following form:

$R_{k}$ : if $X_{1}$ is $A_{1 i}$ and $\ldots$ and $X_{m}$ is $A_{m j}$ then $Z$ is $B_{k}$ with $C F=C F_{k}, X_{1}, X_{m}$ is input variable, $Z$ is output variable, $A_{1 i}, A_{m j}$ is parameterized fuzzy set, $C F_{k}$ is the confidence coefficient of the rule.

The structure of the FNN is shown in Figure 1, and the network will be divided into five levels, and is designed according to the working process of the fuzzy system, and can be a neural network to realize fuzzy inference system.

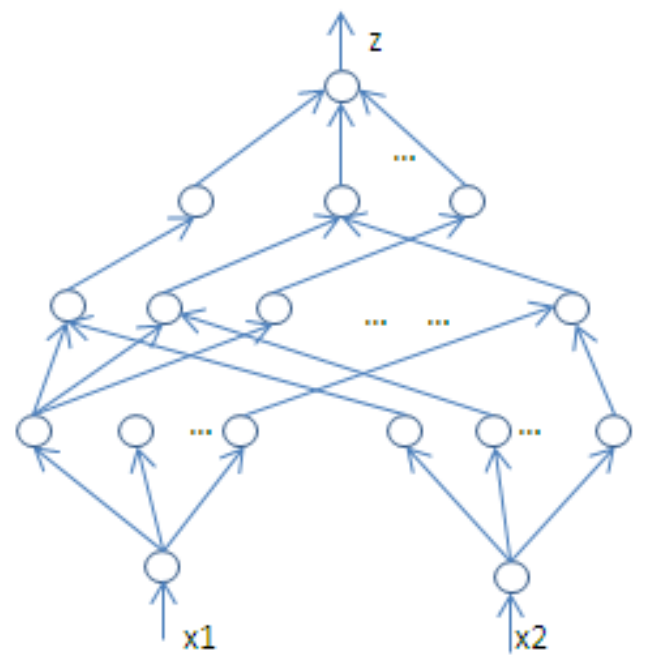

\section{Clarification}

Fuzzy Or

Fuzzy And

Fuzzification

Fig.1. Structure of fuzzy inference system

According to each layer nodes of FNN above defined, the BP learning algorithm is deduced, the error function is:

$E=\frac{1}{2}(t-z)^{2}$

$\delta_{k}$ is defined as:

$\delta_{k}=\frac{\partial E}{\partial z_{k}}=\frac{\partial E}{\partial z} \cdot \frac{\partial z}{\partial z_{k}}=-(t-z) \frac{\alpha_{k}}{\sum_{k} z_{k}}$

Based on the following gradient descent method:

$\frac{\partial E}{\partial w_{k j}}=\frac{\partial E}{\partial z_{k}} \cdot \frac{\partial z_{k}}{\partial w_{k j}}=\delta_{k} \cdot \begin{cases}x_{k j}, & x_{k j} \cdot w_{k j}=M A X_{k} \\ 0, & \text { otherwise }\end{cases}$

Weights can be adjusted for: 


$$
w_{k j}(t+1)=w_{k j}(t)+\Delta w_{k j}=w_{k j}(t)-\beta_{k} \frac{\partial E}{\partial w_{k j}}
$$

Here $\beta_{k}$ is the learning efficiency, the center of the membership functions of output variables also can be adjusted according to the expression:

$$
\begin{aligned}
& \alpha_{k}(t+1)=\alpha_{k}(t)+\Delta \alpha_{k}=\alpha_{k}(t)-\beta_{k} \frac{\partial E}{\partial \alpha_{K}} \\
& \frac{\partial E}{\partial \alpha_{k}}=\frac{\partial E}{\partial z} \cdot \frac{\partial z}{\partial \alpha_{k}}=-(t-z) \frac{z_{k}}{\sum_{k} z_{k}}
\end{aligned}
$$

After the network convergence, the rules can be extracted from the network, and can be simplified. The simplification of the rules is based on the weight of the network. Main idea is to make the rules that network weights is zero or very close to zero deemed invalid rules and removed; in all rules with the same condition, the rules that weights is maximal are retained, moreover all other rules are removed. The size of the initial weights represents the successful probability of the rules. After network training, the probability can be increased or is decreased, that the probability is zero means that the rule no longer exists and should be removed. In order to maintain rules consistency, selecting the rules of maximum probability in the contradictory rules is a good choice.

\section{Experiment and Verification}

With the Kerogen samples data of a certain area as an example, the data in Table 1 is 21 Kerogen samples, in order to verify the effectiveness of the method proposed this paper, the nine samples selected from the Table 1 (select first three samples of each type) form a data table, used for system optimization combination properties and training of FNN, and the rest of the samples is used for inspection.

Tab.1. Kerogen samples data

\begin{tabular}{ccccccccccc}
\hline number & $\mathrm{e}$ & $\mathrm{f}$ & $\mathrm{g}$ & $\mathrm{h}$ & $\mathrm{i}$ & $\mathrm{j}$ & $\mathrm{k}$ & $\mathrm{l}$ & $\mathrm{m}$ & type \\
\hline 1 & 25.0 & 10.0 & 40.0 & 25.0 & 15.2 & 8.13 & 2.54 & 9.1 & 35.8 & 3 \\
2 & 5.0 & 30.0 & 50.0 & 15.0 & 14.3 & 7.55 & 0.79 & 4.4 & 5.6 & 3 \\
3 & 10.0 & 20.0 & 40.0 & 30.0 & 19.3 & 10.18 & 1.93 & 6.2 & 32.1 & 3 \\
4 & 5.0 & 20.0 & 45.0 & 30.0 & 29.5 & 22.00 & 2.14 & 7.2 & 33.1 & 3 \\
5 & 10.0 & 0.0 & 70.0 & 20.0 & 9.4 & 4.04 & 1.17 & 5.6 & 48.4 & 3 \\
6 & 15.0 & 0.0 & 55.0 & 30.0 & 19.5 & 10.49 & 2.01 & 7.6 & 37.9 & 3 \\
7 & 5.0 & 20.0 & 55.0 & 20.0 & 14.2 & 9.50 & 2.66 & 4.3 & 16.2 & 3 \\
8 & 45.0 & 10.0 & 40.0 & 5.0 & 10.1 & 1.55 & 38.54 & 21.9 & 5.7 & 2 \\
9 & 35.0 & 15.0 & 50.0 & 0.0 & 18.0 & 10.79 & 5.68 & 20.3 & 36.9 & 2 \\
10 & 30.0 & 25.0 & 45.0 & 0.0 & 9.8 & 2.35 & 37.60 & 12.2 & 3.2 & 2 \\
11 & 30.0 & 15.0 & 55.0 & 0.0 & 11.0 & 4.54 & 9.55 & 9.4 & 9.9 & 2 \\
12 & 40.0 & 20.0 & 40.0 & 0.0 & 10.0 & 4.67 & 22.74 & 14.6 & 6.4 & 2 \\
13 & 30.0 & 25.0 & 45.0 & 0.0 & 10.6 & 2.62 & 25.61 & 12.8 & 5.0 & 2 \\
14 & 40.0 & 20.0 & 40.0 & 0.0 & 12.8 & 4.50 & 17.22 & 16.5 & 9.5 & 2 \\
15 & 55.0 & 10.0 & 25.0 & 10.0 & 13.1 & 4.95 & 34.08 & 31.4 & 9.2 & 1 \\
16 & 65.0 & 10.0 & 25.0 & 0.0 & 11.6 & 2.41 & 39.29 & 29.9 & 7.6 & 1 \\
17 & 60.0 & 0.0 & 25.0 & 5.0 & 13.1 & 2.09 & 54.80 & 43.7 & 6.8 & 1 \\
18 & 50.0 & 20.0 & 25.0 & 5.0 & 10.9 & 2.43 & 45.13 & 22.6 & 4.9 & 1 \\
19 & 55.0 & 20.0 & 25.0 & 0.0 & 10.0 & 2.10 & 83.85 & 21.9 & 2.6 & 1 \\
20 & 45.0 & 30.0 & 25.0 & 0.0 & 9.1 & 2.93 & 98.54 & 38.2 & 3.5 & 1 \\
21 & 60.0 & 15.0 & 25.0 & 0.0 & 11.8 & 1.78 & 54.82 & 25.2 & 4.6 & 1 \\
\hline
\end{tabular}

Because the rough set theory is only suitable to analysis and process quantified data, therefore, first the Kohonen network algorithm is used to quantify the condition attributes in the data table, in Table 1 each sample corresponds to the nine analysis indicators as condition attributes $C=\{e, f, g, h, i, j, k, l, m\}$, "type" is decision attribute $D=\{d\}$, and the initial rule is discovered from quantified data table based on rough set theory, then FNN training is proceeded on 
these initial rules, after network convergence, the rule was further simplified, and the finally gotten rule set is as shown in Table 2.

Tab.2. Reduced rules set

\begin{tabular}{cccc}
\hline number & $\mathrm{g}$ & $\mathrm{h}$ & $\mathrm{d}$ \\
\hline 1 & 2 & 2 & 3 \\
2 & 2 & 1 & 2 \\
3 & 1 & 1 & 1 \\
\hline
\end{tabular}

According to the fuzzy rules generated, after the other samples were tested and analyzed, the correct rate of classification results is up to $99.5 \%$, but using all the attributes and the same training set, the correct rate of FNN network classification is only $87.3 \%$, at the same time still using the traditional BP network to simulate the above examples, through comparing the classification results, it is found that the classification accuracy of traditional BP network is higher, but will not get better results than the method, and the neural network is a black box, the middle layer nodes and connection weights have no special meaning, so it is difficult for people to understand. In addition, the selection of the node number of middle layer is mainly depended on trial and error, and the improper selection of the node can influence learning speed, and the network training time is longer.

\section{Conclusion}

This paper an integrated method to acquire fuzzy rules is presented, which take full advantage of the Kohonen network, rough sets and fuzzy neural network, so that the integrated method is much better than a single method, and it is verified that the method is an effective method through the example.

\section{References}

[1] FAYYAD UM, PIATETSKY-SHAPIRO G, SMYTH P. Advances in knowledge Discovery and Data Mining [M]. Mass: AAAI/MIT Press, 1996.83-115.

[2] AGRAWAL T, IMIELINSKI R, SWAMI A. Mining association rules between sets of items in large databases[C]//Washington DC: Proceedings of ACM SIGMOD, 1993.

[3] KRYSZKIEWICZ M. Rough set approach to incomplete information system [J]. Journal of Information Science. 1999, 11(3): 271-292.

[4] Shengbing Zhang, Bo Zhang, Dong Cheng. No backtracking algorithm based on rule and database of attribute-oriented induction [J]. Journal of software. 1999,10(7): 506-512.

[5] TAKAGI H. Fusion technology of fuzzy theory and neural networks-survey and future directions [C]// Iizuka, Japan: Pro Int Conf on Fuzzy Logic and Networks. 1990.

[6] PAWLAK Z. Rough sets [J]. International Journal of Computer and Information Sciences. 1982, 11(5): 341-356.

[7] Huanglin Zeng. Rough set theory and its application-new methods of theories about the data [M]. Chongqing: Chongqing university press, 1998.

[8] Zunde Chen, Guangsheng Zhu. Application of Kohonen network to the lateral prediction of oil and gas [J]. Geophysical Prospecting for Petroleum. 1995, 34(2):53-56.

[9] Li Pan, Zuxun Zhang, Jianqing Zhang. Application of rough set in image feature selection [J]. Journal of Data Acquisition and Processing. 2002, 17(1):43.

[10] Xiaohong Lu, Shiquan Chen. Heuristic attribute reduction method based on discernibility matrix and its application [J]. Computer Engineering. 2003, 29(1):56-58.

[11] Yanying Fan and so on. A Complete value reduction algorithm based on rough set theory [J]. Journal of Guangxi Academy of Sciences. 2013, 29(1): 4-6. 